JOURNAL OF APPLIED CRYSTALLOGRAPHY

ISSN 1600-5767

Keywords: book review; lasers.

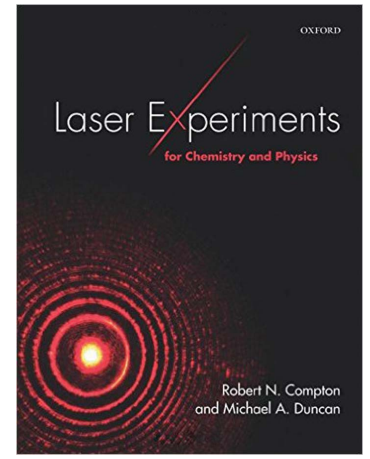

(C) 2016 International Union of Crystallography

\section{Laser Experiments for Chemistry and Physics. By Robert N. Compton and Michael A. Duncan. Oxford University Press, 2015. Pp. 416. Price GBP 35.00 (paper- back, ISBN 9780198742982), GBP 70.00 (hardback, ISBN 9780198742975)}

\author{
Germano Montemezzani*
}

Laboratoire LMOPS, Université de Lorraine et CentraleSupélec, 2 rue E. Belin, F- 57070 Metz, France. *Correspondence
e-mail: germano.montemezzani@univ-lorraine.fr

The importance of lasers in modern science and technology and their (often hidden) role in our everyday life cannot be emphasized enough. While lasers find an enormous number of applications in the industrial, medical and telecommunication sectors just to name a few, they also are an essential tool for fundamental research in physics, chemistry and related areas. While going through experimental scientific literature, one can be surprised by the number of studies that make use of lasers, even in areas one would not expect to.

Laser Experiments for Chemistry and Physics focuses on the use of lasers for fundamental research through laboratory experiments designed for the undergraduate curricula in chemistry and physics. The authors, Robert N. Compton and Michael A. Duncan, are two outstanding authorities in the field of laser-based research in physical chemistry. They build on their respective research backgrounds and on decades of experience with undergraduate laser-based experiments in their laboratories at the University of Tennessee for Compton and at the University of Georgia for Duncan. This is obviously an ideal combination to produce a high-quality and useful book, and in my view the result is well at the level of these expectations. Even though the book is more focused on chemical research (the order of the two disciplines in the title is the right one), physics students and instructors will also find much of interest in this volume.

The book is constructed around 31 chapters divided into five parts, as well as two short appendices and the final index. The first part composed of six chapters gives some background on basic optics and the elementary properties of light (chapters 1 and 2) and on the fundaments of laser physics (chapter 3). It also briefly introduces some laser systems important in the context of the book (chapter 4) and gives a short introduction to nonlinear optics (chapter 5). Chapter 6 describes critical laser safety issues related to eye and skin damage, high voltages, and chemical exposure. It also lists some important recommended laboratory practices. This chapter is particularly welcomed because such contents are rarely found in textbooks. Students in undergraduate classes who encounter lasers and laser-based experiments for the first time will obviously recognize its importance. The six chapters in the introductory section make up a total of 111 pages. They can evidently give only a very basic and necessarily incomplete introduction to the general fields of classical optics, laser physics and nonlinear optics. Even though giving valuable help for the purpose of the experiments described in the book, none of these chapters can replace the reading of one of the many excellent reference textbooks existing for each of these fields.

The core of the book is contained in the 25 chapters, each dedicated to a specific type of experiment, contained in parts II to V, which are entitled Laser experiments for thermodynamics (four chapters), Laser experiments for chemical analysis (three chapters), Laser experiments for quantum chemistry and spectroscopy (14 chapters) and Laser experiments for kinetics (four chapters). The length and the weight given to the individual topics are variable, with chapter lengths ranging from four to 36 pages.

The title of part II is probably a bit more restrictive than its content. One of the chapters is on the determination of the speed of sound in gases, liquids and solids based on the simple measurement of the arrival time of the sound wave triggered by a few nanosecond long laser pulse at another known position. Another chapter describes 
calorimetric measurements by the thermal lensing effect induced by a focused laser on a liquid sample. While these two chapters have an evident relation to thermodynamics, this is less evident for chapter 7, which describes the determination of the speed of light by a simple time-of-flight method, or for chapter 10 describing a laser diffractometer for the determination of the index of refraction.

Part III includes first a chapter on using laser-induced breakdown spectroscopy to obtain a signature of the species contained in a given sample. The experiments described in the following two chapters all employ time-of-flight mass spectrometry as a detection technique. In chapter 12 the ions to be detected in the mass spectrometer are produced by laser desorption. This chapter contains first a useful introduction to time-of-flight mass spectrometry and gives then several examples of mass spectra obtained for different material systems, such as fullerenes, and silver atom, sulfur or phosphorus clusters produced by laser vaporization. Besides a time-of-flight mass spectrometer, these experiments generally require a nanosecond pulsed Nd:YAG laser operated at the second or third harmonic frequency with pulse energies of a few millijoules per pulse. For compounds having higher ionization energies a single photon of the above lasers is not enough for the ionization and a multi-photon process must be used. This is discussed in chapter 13 by the example of the mass spectrometry of metal carbonyls.

Part IV is mainly concerned with spectroscopy and makes up the largest section of the book. It starts with an important and excellent chapter giving the basis of optical spectroscopy, including electronic and rovibrational spectroscopy. It clarifies the various selection rules and gives expressions for the vibrational and rotational energy levels of molecules, taking into account the anharmonicity and the influence of the rotation on the vibrational frequencies. This chapter provides information essential for understanding the spectra discussed in some of the later chapters. The following chapter, 15, is dedicated to quantum chemistry calculations to predict molecular properties by methods now readily available to non-experts and that can be implemented at the undergraduate level on a personal computer. After a short theoretical introduction, the chapter concentrates on the use of the commercial program Gaussian, which is one among the various commercial and open-source programs available for quantum chemistry. The following chapters give specific experiments aimed at various spectroscopic investigations. This includes Raman spectroscopy (chapter 19, Raman spectroscopy under nitrogen; chapter 22, Fermi resonances in $\mathrm{CO}_{2}$; chapter 27, Raman shifting); the resonantly enhanced multiphoton ionization process (chapters 16 and 18) where a tunable laser is needed and the ion signal is measured; electronic absorption spectroscopy by a tunable laser (chapter 17); photo-acoustic spectroscopy (chapter 23); nonlinear optical spectroscopy (chapter 16, third harmonic generation in alkali atoms; chapter 26, vacuum ultraviolet spectroscopy by third harmonic generation in rare gases; chapter 27, stimulated electronic Raman scattering in alkali vapors); and several others. The determination of the dispersion of the circular birefringence in chiral liquids (chapter 20) and the Verdet constant associated with the Faraday effect in $\mathrm{CS}_{2}$ (chapter 21) is also discussed. Despite having various levels of detail, each of these chapters introduces first the theoretical background related to the experiment, which is relevant for general application of the technique. Each chapter subsequently describes the experimental arrangement and gives some examples of results. The undergraduate student will find several practical tips on the experiments, which very rarely find their place in a textbook and almost never in a scientific paper.

The final part, V, contains first a chapter (28) describing the determination of the fluorescence lifetime and fluorescence quenching in iodine vapor. The following chapter uses again Raman spectroscopy, as a function of temperature for conformational analysis of 1,2-dichloroethane. Simple experiments on diffraction of light by blood cells in the Fraunhofer regime are the subject of chapter 30. Finally, the last chapter characterizes the kinetics of the hydrolysis chemical reaction producing glucose and fructose from sucrose in terms of the change of optical activity (circular birefringence) measured in a polarimeter setup. The first appendix gives a list vendors for the equipment needed for the various experiments (lasers, optics, mounts, detectors, electronics, vacuum equipment etc.). Though useful, such lists are necessarily limited and frozen at the moment of the book's publication. Therefore, consulting more complete guides such as the Physics Today Buyer's Guide may still be a good idea. The second short appendix explains some issues related to the measurement of fast signals and will certainly be of interest for young undergraduate students. The final index is reasonably detailed and takes up three pages.

The costs of the laser systems required for the laboratory experiments described in the book are quite variable, which may represent an obstacle for certain undergraduate laboratories in the case of the most costly ones. Such systems include cheap He-Ne lasers, tunable semiconductor diode lasers, pulsed nitrogen lasers, pulsed frequency converted Nd:YAG lasers, and tunable dye lasers or more involved optical parametric oscillators. Other laser systems, such as excimer lasers, may be employed for some of the experiments.

The first edition of a scientific book is rarely free from some small errors or inconsistencies and Laser Experiments for Chemistry and Physics is not an exception. I have detected a certain number of such minor defects of which I give here a selection. Units are not always expressed according to the accepted abbreviation in the International System of units. So, on page 8 one finds ones 'coul' and ' $C$ ' for Coulomb on the same line, as well as 's' and 'sec' for second in two successive lines. In the same line the unit of the vacuum permittivity $\varepsilon_{0}$ lacks an additional metre in the denominator. Some statements in the two optics chapters are too absolute. So, it is not true that the group velocity $v_{\mathrm{g}}$ can always be identified with the speed at which information is carried (page 10) because this would prevent the possibility for $v_{\mathrm{g}}$ to exceed the speed of light in vacuum $c$, which can occur in dissipative media showing anomalous dispersion. Also, it is not true that the 
refractive index $n$ is always larger than or equal to 1 (page 28). On page 37 the term 'optically active' material is used as a synonym of birefringent material (linear birefringence), while usually this term is used only for materials exhibiting a natural polarization rotation and circular birefringence, as used later in the book. The standard abbreviation TEM stands for 'transverse electromagnetic mode' and not for 'transverse electrostatic mode' as stated on page 41. Even though the two inventions are closely related, the Nobel Price for Physics of 2014 was given to Isamu Akasari, Hiroshi Amano and Shuji Nakamura for the invention of blue light-emitting diodes and not for the invention of blue laser diodes, as stated on page 84 . The official citation of the Nobel committe is 'for the invention of efficient blue light-emitting diodes which has enabled bright and energy-saving white light sources'. In the chapter on nonlinear optics it would be better to indicate the polarization induced by the second-order nonlinear optical effect as $P^{(2)}$ and not as $P^{2}$ [equations (5.14)-(5.16)], which may be misunderstood as being the squared polarization. Also, the expression for the phase mismatch $\Delta k$ after equation (5.17) is incorrect. In the chapter on the speed of sound the caption of Fig. 8.6 does not seem to correspond to the figure. On pages 171-172 the mass should be proportional to the square and not to the square root of the time of flight. In the chapter on Faraday rotation, equations (21.10) and (21.20) are the same equation but appear with opposite signs. In the context of this equation it is stated on page 309 that for normal dispersion $\mathrm{d} n /$ $\mathrm{d} v$ is negative, while it is the other way round. I detected almost no typos, showing that evidently a very good proof reading was done. An exception is 'Soliel-Babinet' on page 13, which should read 'Soleil-Babinet'. The use of acronyms is reasonably moderate but could probably be reduced further. For example, on page 9 the acronyms RCP and LCP are defined for, respectively, right and left circular polarization, but just a few lines later the undefined RCPL and LCPL are used.

These minor points do not diminish the value of this carefully written volume, where the description of each laboratory experiment self-consistently includes a theoretical introduction and historical background, experimental details and practical tips, some experimental results, and a discussion, as well as a proper reference list that will help the reader wishing to go deeper into the subject matter. The topics treated in the book are necessarily a subset, the choice of which is evidently influenced by the research and teaching experience of the authors. Therefore instructors and students specially focused in the fields of physical chemistry and spectroscopy will most likely find the highest interest; however, readers interested in broader areas of chemistry and physics should also find this work very useful. If you belong to this potential audience, the purchase of Laser Experiments for Chemistry and Physics will doubtless be a good investment. 